\title{
Studies of Radical Model for Retrieval of Cursive Chinese Handwritten Annotations
}

\author{
Matthew Ma ${ }^{1}$, Chi Zhang $^{2}$, and Patrick Wang, IAPR Fellow ${ }^{3}$ \\ ${ }^{1}$ Panasonic Information and Networking Technologies Laboratory \\ Panasonic Technologies, Inc. Two Research Way, Princeton, NJ 08540 USA \\ ${ }^{2,3}$ College of Computer Science, Northeastern University, Boston, MA 02115
}

\begin{abstract}
Our research focuses on Chinese online ink matching that tries to match handwritten annotations with handwritten queries without attempting to recognize them. Previously, we proposed a semantic matching scheme that uses elastic matching with a dynamic programming approach based on the radical model of Chinese characters. By means of semantic matching, a handwritten annotation may also be retrieved independently of writers via typed text query, or stored texts can be retrieved by handwritten queries. This work concerns with the behavior of the previously proposed radical model in several aspects including character normalization, stroke segmentation, structural information, dynamic programming costs and schemes. Based on our study, a new radical model is proposed. As a result, the recall of retrieval by handwritten query reaches $90 \%$ for the first hit (an improvement of $20 \%$ over previous results) and the recall by text query reaches $80 \%$ when top 20 matches are returned.
\end{abstract}

\section{Introduction and Motivation}

In language computing, both on-line and off-line handwritten Chinese character recognition (HCCR) have been existing for several decades. Although online recognition has the advantage over offline because the temporal order of the input points and strokes is provided, it still has proved to be a more difficult problem than most people anticipated because of the variations of the way people write and a complex training process involved [1]. In addition, a large lexicon is to be incorporated due to the large number of characters $(3,000-5,000)$ that are daily used.

Instead of handwriting recognition, some research work has been conducted on online ink matching that tries to match a handwritten query against raw ink data without attempting to recognize them [4]. This technique can be used in a document annotating and browsing system, which enables users to search their personal notes by a handwritten query. Similar work and various applications also appear elsewhere $[6,7]$.

Recently, a semantic matching method was proposed by Ma et al. [5]. By extending Wang's Learning by Knowledge paradigm [8], this method focuses on the semantic approach that a human learns and recognizes things and realizes such

1 Corresponding author. E-mail: mma@research.panasonic.com 
approach in the matching of Chinese handwritten annotations via a radical model. The semantic matching has several advantages over previous ink matching methods [4]. First, it speeds up the existing ink matching by reducing the size of the problem. For each query, it returns only top candidates based on the matching of radicals that are extracted from handwritten annotations. The traditional raw ink matching is therefore applied only to these top candidates instead of the entire database. Secondly, only a few radicals are used thus the training process is minimized. Third, it enables the user independent retrieval without handwriting recognition. After radicals have been obtained from the raw data strings of one user, another user can type in the query by keyboard, which can be converted to radical codes immediately.

As reported in [5], the incorporation of a semantic model speeds up the matching process significantly. This is done by returning top 30 (out of 200) candidates in our experiments, consequently yielding a reduction of $80 \%$ in computation time. The drawback of semantic matching, however, is that its recall decreased from that of the original raw ink matching due to the low accuracy in radical extraction. The performance of radical extraction also affects the overall recall of retrieving handwritten annotations by typed text queries.

This work is to further study the behavior of semantic model and to improve the online Chinese ink matching results. The proposed study resulted in a new radical model for the matching of Chinese handwritten annotations. The organization of this paper is as follows. Section 2 describes several aspects of structural information in the radical model and the incorporation of such new model in radical extraction. Experiments using our new radical model on the handwritten annotation retrieval are described in Section 3. Finally, conclusions are given in Section 4.

\section{Studies on Radical Model}

The radical model for Chinese language is used to identify known radicals from each handwritten character and utilize these extracted radicals in the retrieval of handwritten annotations. This is called radical extraction. The drawback of the previous radical extraction is its performance. Particularly, the traditional dynamic programming was used without taking into account characteristics of Chinese language. In this section, some new aspects of Chinese radical model will be presented in order to improve the radical extraction performance.

\subsection{Character Normalization and Segmentation}

In this work, we employed some normalization and segmentation techniques, and experiments show they are adequate. 1) Character size normalization maybe possible once characters are successfully segmented. For simplicity, a linear normalization is used. 2) The incoming points, which are usually grouped into strokes based on the online "pen-down" and "pen-up" information, can further be segmented at local minima and maxima of the $y$ values and local minima of the $x$ values. We call these breaking points "internal breaking point". 3) Internal breaking points are further determined whether they are "obscure" or "obvious" depending on the degree of stroke change near it. If the change of strokes is relatively smooth around the internal 
breaking point, this breaking point will be considered "obscure" thus eliminated. 4) In cursive handwriting, sometimes two separate strokes are connected by an extra stroke, i.e. a connection stroke. These extra connections are not random, they are limited only to several types. In reality, the connection stroke " $\boldsymbol{~}$ " may not appear in a handwritten character consistently. The extra connection stroke is more likely to be affected by the speed and direction of the stylus when the character was formed. Therefore, removing this extra connection stroke may reduce the effect on matching between two characters, one with connection strokes and the other without [3].

\subsection{Shape Measurement}

Consider a dynamic programming at stroke level. Let $C=c_{1} c_{2} \ldots c_{m}$ and $R=r_{1} r_{2} \ldots r_{m}$ be stroke sequences for a character and a radical, respectively. The problem of radical extraction is to take a series of operations on sequence $R$, from left to right, and transforms it to a subsequence of $C$. This can be realized by a dynamic programming procedure, in which three basic operations on strokes are defined: (a) insert a stroke, (b) delete a stroke, and (c) substitute a stroke for another. Each operation is associated with a cost. The details of dynamic programming are described elsewhere [4].

In previous implementation, stroke insertion cost and stroke deletion cost are simply in direct proportion to the length of the strokes. As for stroke substitution cost, corresponding points between two strokes are located using a separate dynamic programming procedure on point level, and Euclidean distance between each pair of two points is measured and summed. This method has two disadvantages. First, the dynamic programming on point level is time consuming. Secondly, the Euclidean distances between points can be cumulative.

Ideally, the stroke substitution measures the difference of two strokes, more precisely, the difference of their shapes. However, discrepancies exist in the current

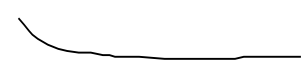

(a) Stroke $\mathrm{s}_{1}$ (b) Stroke $\mathrm{s}_{2}$

$\mathrm{S}_{2}$

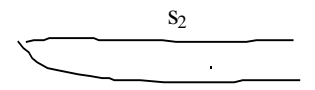

Fig. 1. Discrepancies of substitution cost based on Euclidean distance.

computation scheme. For example, as illustrated in Fig. $1, s_{2}$ is the reference stroke, while $s_{1}$ is the stroke to be compared to $s_{2}$. In the original algorithm, before the substitution cost is computed, each stroke is temporarily shifted so that the top-left corners of the bounding boxes of the strokes are aligned (Fig. 1c). Although $s_{1}$ and $s_{2}$ are overall similar in shape except the beginning part, they will still yield a large Euclidean distance due to the deviation of the beginning part. Therefore, another method for measuring the shape similarity of two strokes based on tangent vectors is proposed. 


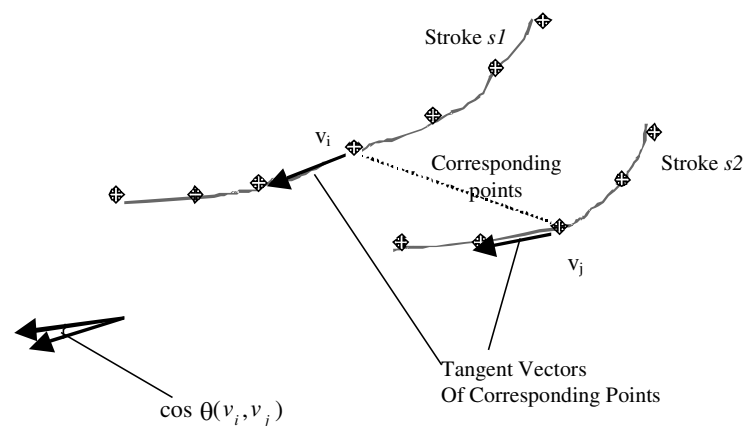

Fig. 2. Illustration of stroke measurement.

Tangent vector at a point of a stroke is defined as the vector from the current point to its next point along the stroke. Referring to Fig. 2, we define the corresponding points of two strokes as follows. Let $s_{1}$ be a stroke with $l_{1}$ points, and $s_{2}$ be a stroke with $l_{2}$ points. $P_{i}$ is the $i$ th point within $s_{1}$, the corresponding point of $P_{i}$ on stroke $s_{2}$ is $P_{j}$, where $j=\left(i / l_{1}\right) l_{2}$. We calculate the substitution cost of two corresponding points $P_{i}$ and $P_{j}$ as follows:

$$
\text { point_sub_cost }\left(P_{i} \mid s_{1}, s_{2}\right)=1-\cos \theta\left(v_{i}, v_{j}\right)
$$

where $v_{i}$ is the tangent vector at point $P_{i}$ and $v_{j}$ is the tangent vector at $P_{j} ; \theta\left(v_{i}, v_{j}\right)$ is the angle between the two vectors, and $\theta \in[0, \pi]$. By summing up the point substitution costs for all the points along the stroke $s_{l}$, we can obtain

$$
\sum_{i=1}^{l} \text { point_sub_cost } t\left(p_{i} \mid s_{1}, s_{2}\right)
$$

stroke_sub_cost $\left(s_{1}, s_{2}\right)$, the substitution cost between stroke $s_{1}$ and $s_{2}$ as:

where $l_{l}$ is the length of stroke $s_{l}$. By further normalizing, we have

$$
\max \left(\frac{l_{2}}{l_{1}}, \frac{l_{1}}{l_{2}}\right) \times \frac{\left(l_{2}+l_{1}\right) / 2}{l_{1}} \sum_{i=1}^{l_{1}} \text { point } \operatorname{sub}_{-} \cos t\left(p_{i} \mid s_{1}, s_{2}\right)
$$

Therefore, we should approximately have

$$
\text { stroke_sub_cost }\left(s_{1}, s_{2}\right) \approx \text { stroke_sub_cost }\left(s_{2}, s_{1}\right)
$$

As can be seen, the new stroke substitution cost can overcome the two disadvantages mentioned earlier. First, by finding the corresponding points, we can eliminate the dynamic programming procedure in finding the pairs of corresponding points. Secondly, the calculation of substitution cost using tangent vectors does not have cumulative effects; therefore, it is a more accurate shape measurement. 


\subsection{Structural Information}

To form a Chinese character, strokes within a character are arranged with some structural relationships (i.e. spatial relationship among strokes). Given a stroke sequence of a character alone without spatial relationships between the strokes, the character can not be determined. In this section, the stroke structural relationships embedded in Chinese language will be studied.

\section{Center Relationships}

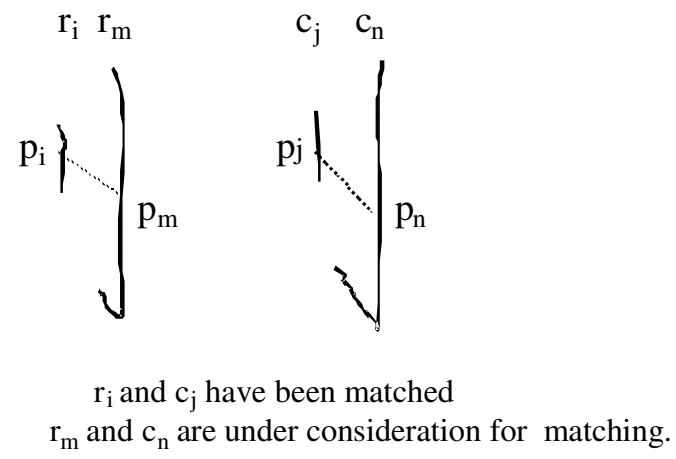

Fig. 3. Illustration of center relationships.

The weighted center of a stroke can be used to indicate the position of a stroke. The structural information can be reflected by the spatial relationship between the two stroke centers. Referring to Fig. 3, let the last two matched (substituted) strokes be $r_{i}$ (the $i$ th stroke of the reference radical) and $c_{j}$ (the $j$ th stroke of the character). The strokes currently under consideration for matching are $r_{m}$ and $c_{n}$. Let $p_{i}, p_{j}, p_{m}, p_{n}$ be the weighted centers for $r_{i}, c_{j}, r_{m}$ and $c_{n}$, respectively. The vector $\overrightarrow{\mathrm{p}_{\mathrm{i}} \mathrm{p}_{\mathrm{m}}}$ reflects the spatial relationship between the two strokes $r_{i}$ and $r_{m}$. Similarly, the vector $\overrightarrow{\mathrm{p}_{\mathrm{j}} \mathrm{p}_{\mathrm{n}}}$ reflects the spatial relationship between $c_{j}$ and $c_{n}$. Before two strokes $r_{m}$ and $c_{n}$ are considered for matching, their spatial relationship with the last two matched strokes are examined:

Rule 1: If $\theta\left(\overrightarrow{\mathrm{p}_{\mathrm{i}} \mathrm{p}_{\mathrm{m}}}, \overrightarrow{\mathrm{p}_{\mathrm{j}} \mathrm{p}_{\mathrm{n}}}\right) \geq \theta_{\mathrm{T}}, r_{m}$ and $c_{n}$ will not be considered for matching. where $\theta_{\mathrm{T}}$ is a threshold, currently set to $\pi / 2$. 


\subsubsection{Starting Point and Ending Point}
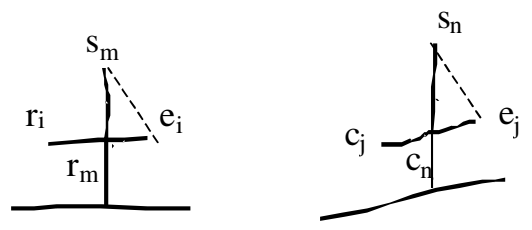

$\mathrm{r}_{\mathrm{i}}$ and $\mathrm{c}_{\mathrm{j}}$ have been matched

$r_{m}$ and $c_{n}$ are under consideration for matching

Fig. 4. Illustration of starting and ending point relationships.

Another important feature that reflects the structure of strokes is the relationship between ending point of a stroke and starting point of the next stroke. As illustrated in Fig. 4, let $e_{i}$ and $e_{j}$ be the ending points of the stroke $r_{i}$ and $c_{j}$, respectively. Let $s_{m}$ and $s_{n}$ be the starting points of the stroke $r_{m}$ and $c_{n}$, respectively. Our criteria is:

Rule 2: If $\theta\left(\overrightarrow{\mathrm{e}_{\mathrm{i}} \mathrm{s}_{\mathrm{m}}}, \overrightarrow{\mathrm{e}_{\mathrm{j}} \mathrm{s}_{\mathrm{n}}}\right) \geq \theta_{\mathrm{T}}$, then $r_{m}$ and $c_{n}$ will not be considered for matching. where $\theta_{\mathrm{T}}$ is a threshold, currently set to $\pi / 2$. Sometimes when two consecutive strokes are connected, the ending point of the first stroke happens to be the starting point of the second stroke, i.e. $e_{i}=s_{m}$ or $e_{j}=s_{n}$. In this case, the above criteria will be ignored and substitution cost for matching shall be calculated.

\subsection{Categorization of Radicals}

In Chinese language, the arrangement of radicals within a character is not random. For example, Cheng et al [1] classified the radical combinations into seven categories such as up-down (UD), left-right (LR) etc. According to the analysis of Lin et al. [2], over $88 \%$ of frequently used Chinese characters belong to the LR and UD types. Based on this, we categorize radicals into two main categories. In the first category, radicals start the first several strokes of a character, while in the second category, radicals end the last several strokes.

The category that a radical belongs is usually known. This category information can be reinforced into our matching process thus a wrong matching will be given a higher cost to prevent it from happening. When a reference radical is matched to a character, penalty will be added if the matched strokes within the character do not fall into the expected category. This is implemented by adjusting the cost in dynamic programming procedure.

If a first category radical is matched to a character, but substitution does not start from the first stroke of the character, all operation cost till the first substitution occurs will also be added to the total cost. Similarly, if a second category radical is not 
matched to the last few strokes of a character, all operation cost from the last substitution occurs till last stroke of the character will be added as penalty.

\subsection{Location Similarity}

By extending from the concept of radical categorization, Ma et al. defined radical profile and location similarity to mathematically represent the location of a radical within a character. In radical extraction scheme, location similarity gives a nonprecise information, or, it can only coarsely confines the location of the radicals [5]. The dynamic programming, however, provides more accurate information of how well radical strokes are matched. We propose to use coarse information (location similarity) to sift out radicals and then use the accurate information (dynamic programming cost) to select and extract the radicals. Once radical candidates with negative location similarity are removed, the remaining radicals are ranked, according to the costs of dynamic programming, and the top two radicals with least costs are chosen as the extracted radicals.

In the previous algorithm, the total dynamic programming cost for matching a reference radical to a part of a character is the sum of all operational costs (insertion, deletion and substitution). Therefore, for each character, when all reference radicals are attempted to match to it, the radicals with fewer strokes tend to yield smaller dynamic programming costs. To solve this, we normalize the total dynamic programming cost by the length of reference radical.

\subsection{Radical Code Evaluation}

After radicals are extracted for each character, a character can be represented by a sequence of radical codes, i.e. radical IDs. When two characters are compared, the matching is performed using dynamic programming on a level of radical codes. Three basic operations are defined: radical insertion, radical deletion and radical substitution, each associated with an operation cost. When evaluating extracted radicals, we propose utilizing the radical extraction cost, which reflects the level of trust for extracted radicals. In implementation, extracted radicals with higher confidence (lower cost) will yield lower radical substitution cost.

\section{Experiments}

Our experimental data consist of three sets: 800 handwritten annotations as reference database (Set I) from four subjects, each writing 200 entries, 800 same handwritten annotations as query database (Set II) from same four subjects (written the second time), and 800 typed text as query database (Set III) corresponding to each of the 800 handwritten annotations. The experiments are conducted in three areas: the radical extraction, which is the core of semantic matching, the overall recall of searching handwritten annotations via handwritten queries and the overall recall of searching by typed text. Three methods are tested and compared with the traditional raw ink elastic 
matching algorithm [4]. $\mathrm{A}_{0}$ represents the original semantic matching algorithm [5]. $\mathrm{A}_{7}$ incorporates the new radical model in our study. $\mathrm{A}_{7}$ ' is almost the same as $\mathrm{A}_{7}$, except that the selection of top candidates returns top 15 matches, instead of top 30 matches.

Table 1 shows the total number of radicals correctly extracted from the query strings and database strings for algorithm $A_{0}$ and $A_{7}$ based on the same reference radical set. As can be seen, as a result of enhanced algorithm $A_{7}$, the number of correctly extracted radicals has increased significantly in compare to the original algorithm. And the performance gain is approximately 2 to 3 times.

Table 1. Comparison of radical extraction rate for algorithm $A_{0}$ and $A_{7}$.

\begin{tabular}{|l|l|l|}
\hline & $A_{0}$ & $A_{7}$ \\
\hline User1 data & 67 & 142 \\
\hline User1 query & 58 & 137 \\
\hline User2 data & 68 & 131 \\
\hline User2 query & 51 & 121 \\
\hline User3 data & 78 & 148 \\
\hline User3 query & 61 & 144 \\
\hline User4 data & 39 & 109 \\
\hline User4 query & 44 & 122 \\
\hline
\end{tabular}

Table 2 lists the recall of the first hits for searching handwriting (Set I) with handwritten queries (Set II). As can be seen, the recall of our new algorithm $A_{7}$ has improved by $20 \%$ for first three users, and $8 \%$ for the $4^{\text {th }}$ user. Moreover, the performance achieved by the new algorithm is very close to that of the traditional elastic matching. To compare $A_{7}$ with traditional elastic matching, because $A_{7}$ returns only top 30 candidates for final matching, it can achieve almost the same performance of original elastic matching while saves computation time by $80 \%$. In algorithm $A_{7}$, we further reduce the computation time in half by returning only 15 top candidates. As a result, the computation time is reduced by more than $90 \%$ of the original elastic matching while achieving comparable results. In fact, it is very interesting to see that for User2, the matching rate of $A_{7}$ is even higher than that of the traditional elastic matching algorithm. The reason is that some interfering candidates for the traditional elastic matching algorithm has been removed from the top candidate selection process.

Table 2. Comparison of recall for first hits (searching handwriting with handwritten queries).

\begin{tabular}{|c|l|l|l|l|}
\hline & $A_{0}$ & $A_{7}$ & $A_{7}{ }^{\prime}$ & $\begin{array}{l}\text { Traditional } \\
\text { Elastic matching }\end{array}$ \\
\hline User1 & 0.725 & 0.885 & 0.845 & 0.895 \\
\hline User2 & 0.755 & 0.92 & 0.91 & 0.88 \\
\hline User3 & 0.77 & 0.96 & 0.94 & 0.98 \\
\hline User4 & 0.805 & 0.865 & 0.855 & 0.88 \\
\hline
\end{tabular}

Figure 5 shows the recall of searching by typed text queries. In this experiment, each handwritten annotation (consisting of a sequence of characters) is converted to a 
sequence of radical codes using radical extraction. When a text query is entered, it is immediately converted to a radical code sequence, then compared with the handwritten annotation database based on radical codes. In Fig. 5, bottom curves in each plot indicate the previous semantic matching results while the top curves stand for $\mathrm{A}_{7}$ results. As can be seen, the matching rate for the first hit is increased by above $100 \%$. Overall, the retrieval rate can reach $60 \%$ with 10 top matches returned and $80 \%$ with 20 top matches returned.

User 1 (Ink vs. Text)
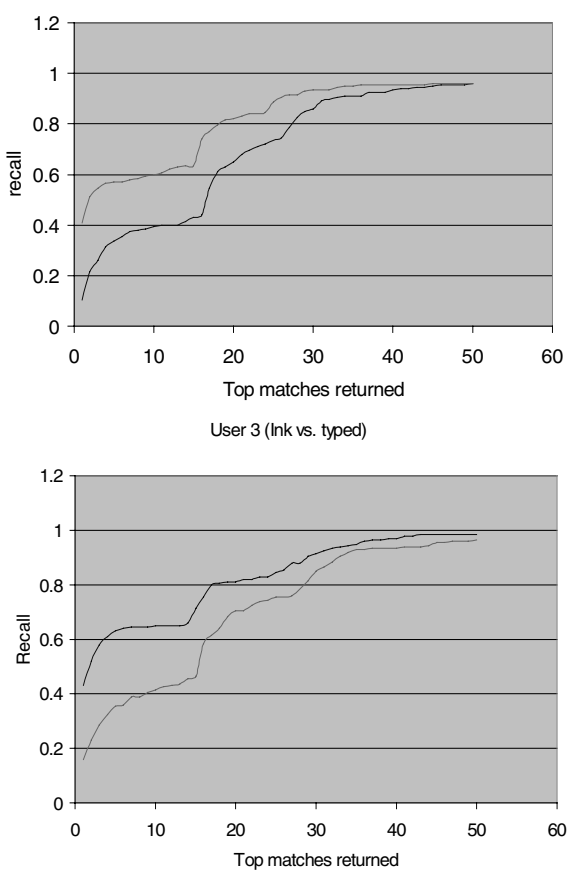

User2 (Ink vs. Ttped)

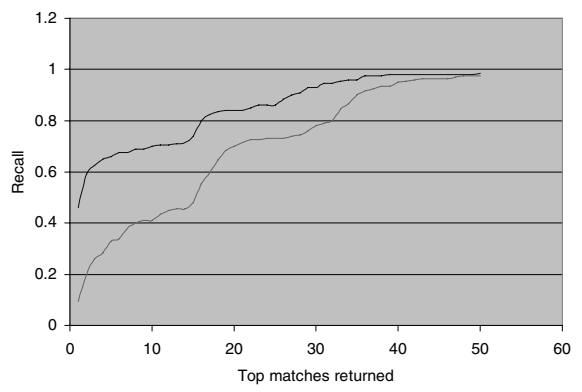

User 4 (Ink vs. typed)

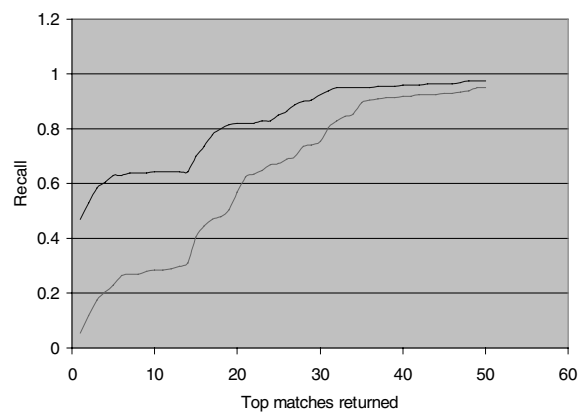

Fig. 5. The recall of searching by typed text queries. The bottom curves in each plot represent the result of $A_{0}$ and the top curves represent the result of $A_{7}$.

\section{Conclusions}

Radical extraction plays an important role in semantic matching, in which semantics in Chinese language are incorporated early into the segmentation of handwritten annotations, and later being used to the matching of handwriting or retrieval of handwriting by typed text queries. In this work, we carefully studied and modified the radical model, based on which the radical extraction rate has increased by $100 \%$ $200 \%$. Several other schemes in the semantic matching network are enhanced. As a result, the recall of searching handwriting by handwritten queries has increased by 
$20 \%$ and reached $90 \%$ for first hits, while the computation time can be reduced by $50 \%$. Moreover, the recall of searching by typed text queries has increased by $100 \%$ for the first hit and reached about $80 \%$ for top 20 matches returned.

The results of this work have shown great potential of semantics in the matching of Chinese handwritten annotations without full bloom handwritten recognition, in which large scale training is usually desired. To conclude, Table 3 illustrates the comparisons between various methods in the searching of Chinese annotations. It is noted that the radical model study in this work may well be extended to other languages or symbols and it is our future work.

Table 3. Comparison of handwriting matching methods.

\begin{tabular}{|l|c|c|c|c|}
\hline & Speed & Performance & $\begin{array}{l}\text { Handwriting } \\
\text { Searchable }\end{array}$ & $\begin{array}{l}\text { Text } \\
\text { Searchable } \\
\text { (user independent) }\end{array}$ \\
\hline $\begin{array}{l}\text { Traditional } \\
\begin{array}{l}\text { Elastic } \\
\text { Matching }\end{array}\end{array}$ & Very slow & Good & Yes & No \\
\hline $\begin{array}{l}\text { Previous } \\
\begin{array}{l}\text { Semantic } \\
\text { Matching }\end{array}\end{array}$ & Fast & Fair & Yes & Promising \\
\hline $\begin{array}{l}\text { New Semantic } \\
\text { Matching }\end{array}$ & Very fast & Nearly good & Yes & Yes \\
\hline
\end{tabular}

\section{Reference}

1. F. Cheng and W. Hsu, Research on Chinese OCR in Taiwan. In Character and Handwriting Recognition, P.S.P. Wang (ed.), pp 139-164. World Scientific, 1991.

2. T.Z. Lin and K.C. Fan, Coarse classification of on-line Chinese characters via structure feature-based method. Pattern Recognition, vol. 27, pp. 1365-1377, 1994.

3. J. Liu, W.K. Cham and M.M.Y. Chang, Stroke order and stroke number free on-line Chinese character recognition using attributed relational graph matching. In Proc. $13^{\text {th }}$ ICPR, pp. 259-263, August, 1996.

4. D. P. Lopresti, M. Y. Ma, P. S. P. Wang and J.D.Crisman, Ink matching of cursive Chinese handwritten annotations, Int. J. of Pattern Recognition and Artificial Intelligence, Vol.12, No.1, pp.119-141, 1998.

5. M. Y. Ma and P. S. P. Wang, Using semantics in matching cursive Chinese handwritten annotations, In Proc. SSPR'98, pp.292-301, 1998.

6. R. Manmatha, C. Han, E. M. Riseman and W. B. Croft, Indexing Handwriting Using Word Matching, Proc. of the first ACM Intl. Conf. on Digital Libraries, pp 151-159, 1996.

7. A. Poon, K. Weber and T. Cass, Scribbler: A Tool for Searching Digital Ink, Companion Proceedings of the CHI, pp. 252-253, 1995.

8. P. S. P. Wang, Learning, Representation, understanding and recognition of words - An intelligent approach, In Fundamentals in Handwriting Recognition. S. Impedovo (ed.), pp. 81-112, Springer-Verlag, 1994.

9. X.-H Xiao and R.-W Dai. Online handwritten Chinese character recognition directed by components with dynamic templates. In Proc. $17^{\text {th }}$ Int. Conf. On Comp. Proc. Of Oriental Lang., pp 89-94, Hong Kong, April 1997. 